\title{
Avaliação da dose de radiação ocupacional de extremidades em exame de histerossalpingografia
}

\author{
D. Filipov ${ }^{\text {a }}$ S. T. A. Kotowski ${ }^{\text {a }}$ H. R. Schelin ${ }^{\text {b }}$ \\ ${ }^{a}$ Departamento Acadêmico de Física, Universidade Tecnológica Federal do Paraná, \\ 80230-901, Curitiba-PR, Brasil \\ ${ }^{b}$ Instituto de Pesquisa Pelé Pequeno Príncipe, \\ 80250-200, Curitiba-PR, Brasil \\ danifilipov@gmail.com
}

\section{RESUMO}

No exame de Histerossalpingografia (HSG) sempre há uma profissional presente com suas mãos muito próximas do campo de radiação. Com base na regulamentação 3.01 da CNEN, indivíduos ocupacionalmente expostos (IOEs) à radiação possuem valores limites de dose equivalente para as extremidades de $500 \mathrm{mSv} / \mathrm{ano}$. Sendo assim, o presente estudo tem como objetivos verificar a dose equivalente na região das mãos de um IOE que realiza o exame de HSG e compará-la com o limite da CNEN para IOEs e com estudos similares. Foi utilizado um phantom humanoide para simular a paciente e uma câmara de ionização, a qual foi posicionada no local comumente ocupado pelas mãos da profissional. O resultado da dose equivalente na região das mãos ( $30 \mathrm{mSv} / \mathrm{ano})$ equivale a $6 \%$ do limite da dose anual da CNEN para IOEs, mas é próximo à maior parte dos estudos feitos com fluoroscópios. Sendo assim, a otimização da proteção radiológica se faz necessária para se reduzir esses resultados.

Palavras-chave: histerossalpingografia, dosimetria ocupacional, proteção radiológica.

ISSN: 2319-0612

Accepted 2018-07-16 


\begin{abstract}
In the Hysterosalpingography (HSG) exam there is always a professional present with their hands very close to the radiation field. Based on the 3.01 CNEN regulation, individuals occupationally exposed (IOEs) to radiation have equivalent dose limit values for the extremities of $500 \mathrm{mSv} / \mathrm{year}$. Thus, the present study aims to verify the equivalent dose in the region of the hands of a professional that performs the HSG procedure and compare it with the CNEN limit to the IOEs and with similar studies. A humanoid phantom to simulate the patient and an ionization chamber, which was placed in the place commonly occupied by the professional hands, were used. The equivalent hand dose result ( $30 \mathrm{mSv} /$ year) equals $6 \%$ of CNEN's annual dose limit to the IOE, but is close to most studies using fluoroscopes. Therefore, the optimization of radiological protection is necessary to reduce these results.
\end{abstract}

Keywords: hysterosalpingography, occupational dosimetry, radiological protection.

\title{
1. INTRODUÇÃO
}

Um procedimento comumente realizado em radiologia e que expõe à radiação ocupacionalmente alguns indivíduos é o exame de histerossalpingografia (HSG), o qual consiste na demonstração radiográfica do trato reprodutivo feminino afim de se estudar, principalmente, distúrbios da fertilidade da paciente. E, durante todo o procedimento, sempre há uma profissional dentro da sala de exames, com as mãos muito próximas do raio central [1,2].

Para que a dose de radiação em certas regiões de um indivíduo não seja elevada, existem alguns princípios básicos de proteção radiológica, sendo um deles o da limitação da dose individual. Através desse princípio, limitam-se valores de dose efetiva ou de dose equivalente para trabalhadores e indivíduos do público. No caso das extremidades de um(a) profissional, o limite da dose equivalente é de $500 \mathrm{mSv} / \mathrm{ano}$, de acordo com a publicação 3.01 da CNEN (Comissão Nacional de Energia Nuclear) [3].

Alguns estudos já foram realizados em exames de HSG [4-6], de forma que os objetivos do presente estudo são estimar a dose equivalente na região das mãos de uma profissional que a- 
companha o exame e comparar os resultados obtidos com o limite de dose na região das extremidades e com os estudos similares.

\section{MATERIAIS E MÉTODOS}

Foi utilizado um phantom humanoide (manequim preenchido com água) para simular uma paciente adulta que realiza o exame de HSG.

Para se verificar a dose de radiação na região das mãos da profissional, uma câmara de ionização de placas paralelas foi posicionada no local que representa a superfície das mãos da trabalhadora (a $\sim 25 \mathrm{~cm}$ do raio central). A Figura 1 mostra a montagem do experimento.

Figura 1 - A) Identificação da distância da posição das mãos do profissional em relação ao raio central do exame; B) Posição da câmara de ionização no local identificado na imagem “A”; C) Visão do phantom com a câmara de ionização.

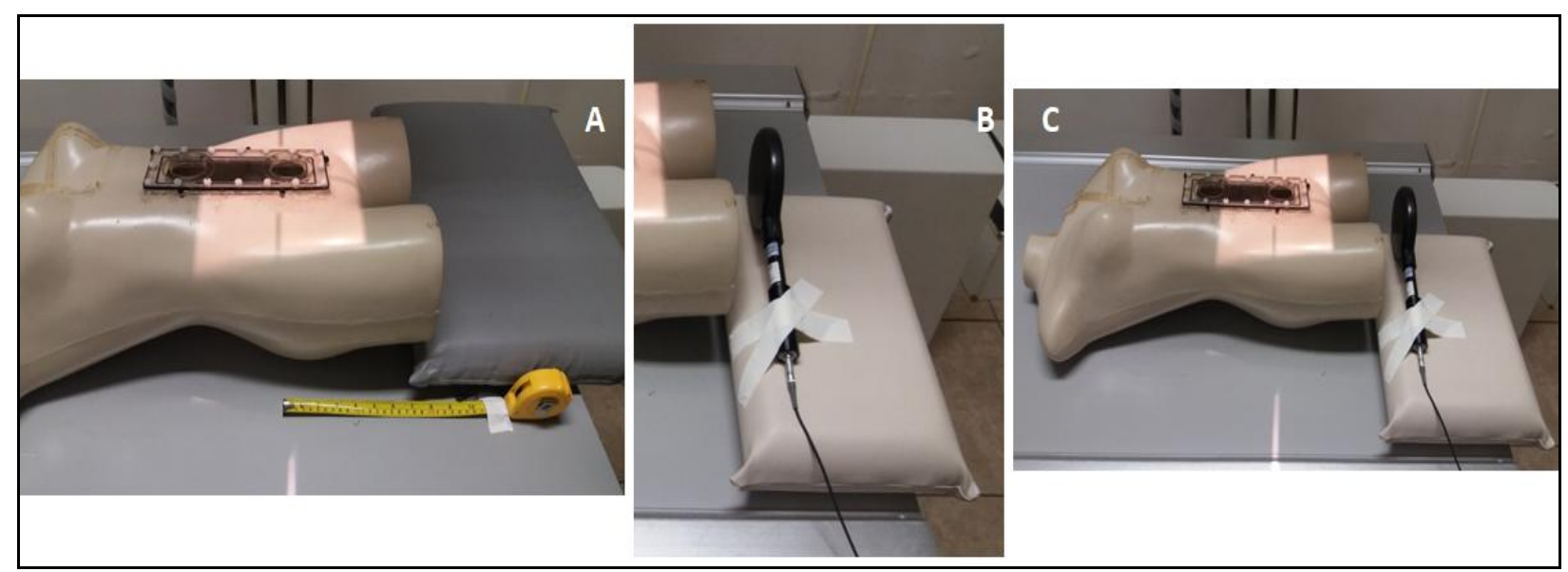

A câmara de ionização empregada é da marca Radcal, modelo 10X6-180 com $180 \mathrm{~cm}^{3}$ de volume sensível. Ela foi calibrada no LMRI do DEN da UFPE, cujo certificado de calibração é o 1036RX/0317.

O exame de HSG foi simulado, no equipamento Silhouette VR (General Electric), com as duas técnicas comumente empregadas na clínica estudada, considerando-se uma distância focofilme de $100 \mathrm{~cm}$ e um total de 8 radiografias realizadas: $70 \mathrm{kVp}$ com $40 \mathrm{mAs}$ e $80 \mathrm{kVp}$ com $32 \mathrm{mAs}$. 


\section{RESULTADOS E DISCUSSÕES}

A Tabela 1 mostra as estimativas da dose equivalente total na região das mãos da profissional, para as duas técnicas empregadas, além dos dados de estudos similares.

Tabela 1: Parâmetros técnicos do exame de HSG e doses equivalentes do presente estudo e de outras pesquisas similares.

\begin{tabular}{|c|c|c|c|}
\hline \multirow{2}{*}{ Estudos } & \multirow{2}{*}{ Técnica } & \multicolumn{2}{|c|}{ Dose Equivalente (mSv) } \\
\hline & & Por exame & Por ano \\
\hline \multirow{2}{*}{ Presente } & $70 \mathrm{kVp}, 40 \mathrm{mAs}, \mathrm{DFoFi}=1 \mathrm{~m}, 8$ imagens & $0,32000 \pm 0,00002$ & $27,0000 \pm 0,0014 * *$ \\
\hline & $80 \mathrm{kVp}, 32 \mathrm{mAs}, \mathrm{DFoFi}=1 \mathrm{~m}, 8$ imagens & $0,39000 \pm 0,00005$ & $33,0000 \pm 0,0045 * *$ \\
\hline [4] & 90-99 kVp, 40-50 mAs, 3 imagens * & 0,62 & 191 \\
\hline [5] & $9 \pm 2$ imagens, $2 \pm 1$ minuto de exposição $*$ & 0,2 & 22 \\
\hline [6] & 43 s de exposição * & 0,2 & 21 \\
\hline
\end{tabular}

Nota: * Parâmetros em aparelhos de fluoroscopia; ** São realizados, em média, 84 exames de HSG por ano no local onde foi realizada a pesquisa. Dessa forma, os valores médios de dose verificados por exame foram multiplicados por 84 , para se chegar à dose anual.

Os estudos foram analisados e verificou-se que os mesmos monitoraram os profissionais através do uso se dosímetros termoluminescentes (TLD - thermoluminescent dosimeters) diretamente nos indivíduos, enquanto que o presente estudo utilizou uma câmara de ionização que é adequada para a dosimetria de baixas energias.

Contudo, a grande dimensão da câmara faz com que os valores de dose verificados sejam compatíveis aos de uma área e não de um único ponto. De qualquer forma, o resultado verificado ( 30,0000 $\pm 0,0033 \mathrm{mSv} / \mathrm{ano})$ não superou o limite anual para as extremidades dos profissionais, de $500 \mathrm{mSv} / a n o$ (de acordo com a regulamentação 3.01 da CNEN [3]) e caso as medidas fossem realizadas com TLDs os resultados seriam ainda menores.

Entretanto, ao se reduzir esses resultados, os mesmos estarão próximos aos dos artigos [5] e [6], ambos realizados com fluorosocópios (os quais empregam altos tempos de exposição).

Logo, sugere-se à clínica deste estudo o uso de técnicas de otimização para que tais resultados sejam devidamente reduzidos. 


\section{CONCLUSÕES}

Os objetivos deste estudo eram estimar a dose equivalente na região das mãos de uma profissional que acompanha o exame de HSG e comparar os resultados com o limite de dose na região e com os estudos similares.

Verificou-se que a dose média nesta região é $\sim 6 \%$ do limite de dose para as extremidades e foi superior à maior parte dos resultados de outros artigos, provavelmente, devido à metodologia empregada. Entretanto, caso as medidas fossem realizadas com outros detectores, verificar-seiam dados próximos a estudos realizados com fluorosocópios.

Assim, técnicas de otimização são sugeridas à clínica para que tais valores de dose sejam devidamente reduzidos.

\section{REFERÊNCIAS}

[1]. BOnTRAGER, K. L. Tratado de Técnica Radiológica e Base Anatômica. $7^{\mathrm{a}}$ edição, Rio de Janeiro: Elsevier Editora Ltda, 2010.

[2]. In: BUSHONG, S. C. Radiologic science for technologists: physics, biology and protection, $6^{\text {th }}$ ed., St.Louis: Mosby, 1997. p. 430-493.

[3]. ICRP - International Commission on Radiological Protection. Recommendations of the International Commission on Radiological Protection. ICRP Publication 103 Ann, 2007, vol. 37 , pp. 1-332.

[4]. JIBIRI, N. N., et al, Occupational Radiation Exposure to the Extremities of Medical Staff during Hysterosalpingography and Radionuclide Bone Scan Procedures in Several Nigerian Hospitals. Mol Imaging Radionucl Ther, v. 25, p. 114-120, 2016. 
[5]. CANEVARO, L.V., DIAS RODRIGUES, B.B., MAURÍCIO, C.L.P. Radiation exposure of patients and workers during histerosalpingography, In: 12TH INTERNATIONAL CONGRESS OF INTERNATIONAL RADIATION PROTECTION ASSOCIATION, 2008, Buenos Aires, Argentina.

[6]. BULS, N., OSTEAUX, M. Patient and staff dose during hysterosalpingography. In: INTERNATIONAL CONFERENCE ON RADIOLOGICAL PROTECTION OF PATIENTS IN DIAGNOSTIC AND INTERVENTIONAL RADIOLOGY, NUCLEAR MEDICINE AND RADIOTHERAPY, 2001, Malaga, Espanha. 